\title{
Alpha-generation as basic response-signature to transcranial magnetic stimulation (TMS) targeting the human resting motor cortex: A TMS/EEG co-registration study
}

\author{
DOMENICA VENIERO, ${ }^{\mathrm{a}, \mathrm{b}}$ DEBORA BRIGNANI, ${ }^{\mathrm{a}}$ GREGOR THUT, ${ }^{\mathrm{c}}$ AND CARLO MINIUSSI ${ }^{\mathrm{a}, \mathrm{b}}$ \\ ${ }^{a}$ Cognitive Neuroscience Section, IRCCS San Giovanni di Dio Fatebenefratelli, Brescia, Italy \\ ${ }^{\mathrm{b}}$ Department of Biomedical Sciences and Biotechnology, National Institute of Neuroscience, University of Brescia, Brescia, Italy \\ ${ }^{\mathrm{c}}$ Centre for Cognitive Neuroimaging, Institute of Neuroscience and Psychology, University of Glasgow, Glasgow, UK
}

\begin{abstract}
The effects of repetitive transcranial magnetic stimulation (rTMS) on cortical excitability are usually inferred from indirect indexes, such as EMG responses. It has now become possible to directly evaluate rTMS impact by means of concurrent EEG recording. The aim of this study was to examine the modulation induced by high frequency rTMS (20 $\mathrm{Hz}$ ) over left primary motor cortex on the ongoing oscillatory activity. Thirteen subjects underwent two sham and a real rTMS session while acquiring EEG. Event-related desynchronization/synchronization was calculated for the $\alpha$ and $\beta$ bands. rTMS induced a dose-dependent increase in synchronization in both bands over central and parietal sites. The strongest effect found for the $\alpha$ band outlasted the end of the stimulation. Considering previous studies, our data suggest that $\alpha$ generation may represent an intrinsic induced response and a basic response signature to TMS targeting the human resting motor cortex.
\end{abstract}

Descriptors: ERD/ERS, TMS/EEG co-registration, Alpha band, TMS-evoked potentials

The popularity of repetitive transcranial magnetic stimulation (rTMS) is due to its potential to promote changes in cortical excitability that may outlast the end of the stimulation. Despite the lack of knowledge about its mechanisms of action, a number of studies indicate that the direction and duration of rTMS effects depend on number of pulses, intensity, and, above all, on the stimulation frequency. In regards to the latter, a dichotomy between high frequency rTMS $(\geq 5 \mathrm{~Hz})$ and low frequency rTMS $(\leq 1 \mathrm{~Hz})$ is widely accepted (Fitzgerald, Fountain, \& Daskalakis, 2006). This distinction is based on physiological studies performed over primary motor cortex (MI), reporting an increase or a decrease of motor-evoked potential (MEP) amplitude when applying high or low frequency stimulation, respectively. The induced modulations on peripheral measurements have been interpreted as indexing a concurrent local increase or decrease of excitability in the stimulated cortex (Chen et al., 1997; Maeda, Keenan, Tormos, Topka, \& Pascual-Leone, 2000; Pascual-Leone, Valls-Sole, Wassermann, \& Hallett, 1994) and the basic idea has been generalized to nonmotor cortices. This possibility of achieving differential changes in brain activity with rTMS has important implications for clinical therapeutics in various neurological and psychiatric disorders. However, more

Address correspondence to: Carlo Miniussi, Dipartimento di Scienze Biomediche e Biotecnologie, Università di Brescia, Viale Europa 11, 25123 Brescia, Italy. E-mail: miniussi@med.unibs.it recent reports describe the modulatory influence of rTMS as more complex than it is classically defined, highlighting that the distinction between inhibitory or facilitatory outcome may be too reductive (Houdayer et al., 2008; Miniussi et al., 2008).

In opposition to classic motor cortex rTMS studies, which inferred cortical modulations from peripheral measurements (Maeda et al., 2000; Pascual-Leone et al., 1994), new ways for characterizing TMS impact on brain activity are provided by recently introduced approaches aimed at recording the direct cortical responses. The combination of TMS with other neuroimaging techniques, such as functional magnetic resonance imaging, positron emission tomography, or electroencephalogram (EEG) provide an assessment of how TMS affects neural processing both locally and in remote interconnected brain regions (Bestmann et al., 2008; Komssi \& Kahkonen, 2006; Miniussi \& Thut, 2010; Paus, 2005; Siebner et al., 2009). The associated evidence has suggested that the effects induced by several off-line rTMS protocols were site specific, but not site limited. Among these combinations, TMS-EEG has proven to be a versatile approach to reveal TMS effects on brain activity (Ferreri et al., 2010; Komssi, Savolainen, Heiskala, \& Kahkonen, 2007). In addition to TMS-evoked potentials (TEPs), the reactivity of brain areas can be further approached from the perspective of TMS-evoked oscillatory activity or induced changes to ongoing oscillations, parts of which are thought to reflect the activity or excitability state of cortico-cortical or cortico-thalamic networks. In this vein, TMS-EEG coregistration offers the possibility of defining 
what kind of neuronal oscillations are triggered by TMS in a resting brain, and to link them to behavioral measurements (for a review, see Thut $\&$ Miniussi, 2009). Moreover, studying the time course of the oscillatory modulation could be an alternative way of assessing the duration of rTMS effects (Thut \& Pascual-Leone, 2010), traditionally tested by collecting MEPs amplitude at different time points after rTMS application (Maeda et al., 2000; Pascual-Leone et al., 1994)

Only few studies have investigated how TMS can modulate ongoing oscillatory activity, when delivered over MI. Considering those studies in which the subject was in a resting condition, TMSinduced oscillations have been recorded after single pulse, as well as low and high frequency stimulation. Single pulse TMS has been shown to induce an intensity-dependent synchronization in the $\alpha$ and $\beta$ bands. The increased power was short-lived and topographically restricted to brain areas close to the hot spot (Fuggetta, Fiaschi, \& Manganotti, 2005; Paus, Sipila, \& Strafella, 2001). More recently, two studies explored the effect of rTMS over MI, mainly focusing on $\alpha$ and $\beta$ event-related desynchronization/synchronization (ERD/ERS). Brignani, Manganotti, Rossini, and Miniussi (2008) applied TMS at $1 \mathrm{~Hz}$ frequency and found a dosedependent increase in power of oscillatory activity, reaching larger amplitude for the $\alpha$ compared to the $\beta$ band. The topography of $\beta$ induction showed a focal effect over central region, whereas the $\alpha$ band power increase, initially restricted to the central hot-spot, spread to ipsilateral parietal sites. In line with these results, Fuggetta, Pavone, Fiaschi, and Manganotti (2008) also reported that $5 \mathrm{~Hz}$ rTMS induces an increase in $\alpha$ and $\beta$ power, although the effects were short-lived, being absent after the end of the train. The topographical distribution of TMS effect was maximal over the stimulated cortex, with some spreading towards parietal sites. In summary, when TMS was applied over primary motor cortex and its outcome was assessed by means of power changes in resting conditions, a strong $\alpha$ and a weaker $\beta$ induction over central and parietal leads have been found, regardless of the protocol (single pulse, low or high frequency TMS).

Our working hypothesis, therefore, was that $\alpha$ and co-occurring $\beta$ induction may represent a basic response-signature to TMS over MI, and consequently that their modulation does not follow the classical dichotomy between low versus high frequency rTMS. Alternatively, it could be that the high frequency stimulation of $5 \mathrm{~Hz}$ chosen by Fuggetta et al. (2008) was not high enough to also reveal a dichotomy relative to the effects of $1 \mathrm{~Hz}$ stimulation by Brignani et al. (2008). Therefore, we here set the stimulation frequency at $20 \mathrm{~Hz}$, clearly higher than the $5 \mathrm{~Hz}$ frequency previously applied (Fuggetta et al., 2008). If our working hypothesis were correct, TMS at $20 \mathrm{~Hz}$ should induce $\alpha$ and $\beta$ increases identical to all other protocols.

Analogous to previous work (Brignani et al., 2008; Fuggetta et al., 2005, 2008), we probed dose-dependent effects of $20 \mathrm{~Hz}$ rTMS over M1 through focusing on central $\alpha$ and $\beta$ bands thought to reflect typical motor cortex rhythms, as suggested by source estimates (Hari \& Salmelin, 1997) and their modulation during sensorimotor processing (Neuper, Wortz, \& Pfurtscheller, 2006). To further facilitate comparison with previous studies (Brignani et al., 2008; Fuggetta et al., 2008), we adopted the same analysis approach, consisting of ERD/ERS calculation, which expresses changes of EEG power in a specific frequency band recorded during an interval of interest in percentage relative to the power of the same frequency band in a reference (baseline) period (Pfurtscheller \& Aranibar, 1977). In our design, each experimental session started with sham stimulation, representing the reference period. The real stimulation following sham represented the interval of interest. Moreover, to examine whether the induced changes in EEG power outlasted the real stimulation, a second sham session was carried out (i.e., Post-real stimulation) representing the second period of interest, again referenced to the first sham session.

\section{Materials and Methods}

\section{Participants}

Thirteen healthy, right-handed volunteers ( 8 female, mean age: $21.8 \pm 3$ years) participated in this study after giving their written informed consent. None of the participants had any contraindication to rTMS or any neurological, psychiatric, or other relevant medical problems (Rossi, Hallett, Rossini, \& PascualLeone, 2009). The protocol was performed in accordance with ethical standards and was approved by the Ethical Committee at the IRCCS San Giovanni di Dio Fatebenefratelli.

\section{Procedure}

Participants were seated in a comfortable armchair in a dimly illuminated, electrically shielded, and sound-proof room with their hands pronated in a relaxed position. During the experiments, subjects were required to focus on a central fixation point. Two sessions of sham magnetic stimuli (Sham1, i.e., reference and Sham2, i.e., Post-real) interleaved with one session of magnetic stimulation (Real) were delivered. The entire experimental session lasted $\sim 80 \mathrm{~min}$ (mounting of electrodes included).

\section{Transcranial Magnetic Stimulation}

TMS pulses were delivered using a SuperRapid transcranial magnetic stimulator connected to four booster modules and a double $70 \mathrm{~mm}$ standard figure-of-eight coil (Magstim Company, Whitland, UK) that generates $2.2 \mathrm{~T}$ as a maximum stimulator output. The coil was placed tangentially to the scalp with the handle pointing backwards and laterally at about a $45^{\circ}$ angle away from the midline, approximately perpendicular to central sulcus. Each experimental session started with the coil positioning. In order to find the motor hot-spot, the coil was moved in steps of about $0.5 \mathrm{~cm}$ in the fronto-central region of the scalp. The hot-spot was defined as the point where TMS induced the maximum MEP from the relaxed abductor digiti minimi (ADM) muscle of the right hand. After the target area was found, the coil was stabilized in the same position by means of a mechanical support that consisted of an aluminium holding arm (Magic Arm, www.Manfrotto.com, with two large clamps) and a heavyduty tripod. Once the coil was immobilized, the resting motor threshold (RMT) was determined, defined as the lowest stimulus intensity, which produced in the ADM muscle at least five MEPs of $50 \mu \mathrm{V}$ out of 10 consecutive stimuli (Rossini et al., 1994).

Each participant underwent three 10-min sessions of rTMS: a sham-TMS reference session (Sham1) followed by real-TMS (Real) and a second Post-real sham-TMS session (Sham2), separated by a TMS-free interval of $2 \mathrm{~min}$ to allow coil replacement. Sham stimulations were performed with the real coil turned over and a $30 \mathrm{~mm}$-thick plywood shield (of the same shape and size as the coil) fastened to the coil and placed against the electrodes 
(Harris \& Miniussi, 2003). The compact wood acts as a physical spacer reducing the induced electric field, being roughly 8-fold lower than under real stimulation (Rossi et al., 2007). Magnetic stimuli were delivered at $100 \%$ of RMT over left MI at $20 \mathrm{~Hz}$ repetition rate, so that a total of 400 stimuli were divided in 40 trains of 10 stimuli ( 0.45 -s train duration), separated by an intertrain interval of 14.55-s duration (see Figure 1). For practical reasons, the stimulation was limited to the left M1. From a physiological and anatomical point of view, left and right hemispheric motor cortices are substantially equivalent and should not differ in terms of rTMS effect direction. Some TMS studies reported a lower motor threshold for the activation of dominant hand muscles, which represents the state of the cortico-spinal tract, whereas no difference has been reported in terms of cortical excitability, for example, tested by paired pulse techniques (Macdonell et al., 1991; Triggs, Calvanio, Macdonell, Cros, \& Chiappa, 1994). Moreover, a TMS/EEG study on TMS-evoked potentials confirmed that TMS stimulation at different intensity evokes similar responses over left and right M1 (Komssi, Kahkonen, \& Ilmoniemi, 2004).

\section{EEG and MEP Recordings}

TMS-compatible EEG equipment (BrainAmp 32 MR plus, BrainProducts $\mathrm{GmbH}$, Munich, Germany) was used for recording EEG from the scalp (Bonato, Miniussi, \& Rossini, 2006; Veniero, Bortoletto, \& Miniussi, 2009). The EEG was contin- uously acquired from 29 recording sites (Fp1, Fp2, F7, F3, Fz, F4, F8, FC5, FC1, FC2, FC6, T7, C3, Cz, C4, T8, CP5, CP1, CP2, CP6, P7, P3, Pz, P4, P8, O1, Oz, O2, and Iz) using electrodes mounted on an elastic cap. Additional electrodes were used as ground and reference. The ground electrode was positioned over Fpz, while mastoids served as the active reference for all electrodes. MEPs were collected from right and left ADM via surface electrodes in belly tendon montage. The signal was band pass filtered at $0.1-$ $1000 \mathrm{~Hz}$ and digitized at a sampling rate of $5000 \mathrm{~Hz}$. In order to minimize overheating of the electrodes proximal to the stimulating coil, TMS-compatible $\mathrm{Ag} / \mathrm{AgCl}$ sintered ring electrodes were used. Skin/electrode impedance was maintained below $5 \mathrm{k} \Omega$ for cortical and electromyogram recordings. Horizontal and vertical eye movements were detected by recording the electrooculogram (EOG). The voltage between two electrodes located to the left and right of the external canthi recorded horizontal eye movements. The voltage between reference electrodes and electrodes located beneath the right eye recorded vertical eye movements and blinks. In order to reduce auditory contamination of EEG induced by coil clicks, white noise (about $90 \mathrm{~dB}$ ) was played through insert earphones during the entire experiment.

\section{EEG Analysis}

Analagous to previous TMS/EEG studies focusing on ERD/ERS (Brignani et al., 2008; Fuggetta et al., 2008), $\alpha$ and

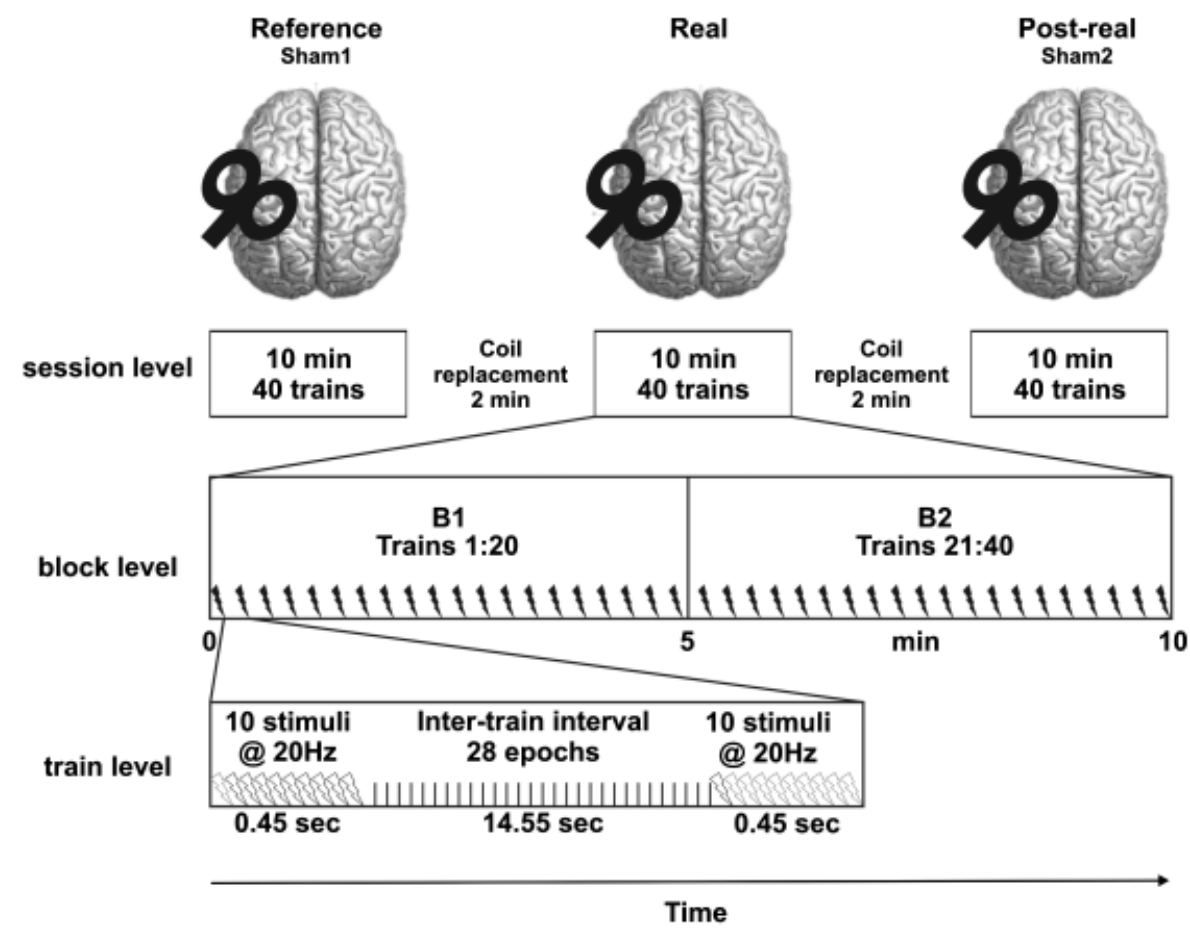

Figure 1. Stimulation protocol and schematic representation of event-related desynchronization/synchronization (ERD/ERS) analysis. Session level: The experiment consisted of three successive sessions starting with a sham stimulation, followed by a real and then a final sham stimulation (respectively, Sham1, Real, and Sham2). Sham stimulation was used throughout, instead of pre- and post-TMS baseline recordings, to equalize TMS-unspecific effects over the experiment (e.g., coil-clicks on vigilance). Each session lasted $10 \mathrm{~min}$, during which 40 TMS trains were delivered at individual resting motor threshold. Block level: To investigate the ERD/ERS modulations over time within a session, the 40 trains were divided in two series of 20 trains (B1- block 1 and B2-block 2), corresponding to 5 min of stimulation each. Train level: Each train consisted of 10 stimuli delivered at $20 \mathrm{~Hz}$ and was followed by an intertrain interval of $14.55 \mathrm{~s}$. Each intertrain interval was divided in 28 epochs of $500 \mathrm{~ms}$, excluding the first $500 \mathrm{~ms}$ after train-offset. The mean ERD/ERS induced during B1 and B2 resulted from the average of the 28 epochs obtained from each intertrain interval. TMS-specific effects were calculated by referring induced $\alpha$ and $\beta$ power (correcting ERD/ERS-measures) during Real to the Sham1-control (Reference). Their time-course after real stimulation was assessed by expressing changes in the same bands calculated during Sham2 (Post-real) and referred to the Sham1-control (Reference). 
$\beta$ modulations induced by rTMS were processed for nine electrodes (F3, Fz, F4, C3, Cz, C4, P3, Pz, and P4) off-line to recordings using Scan 4.3 (Compumedics Neuroscan) and EEGLAB (Delorme \& Makeig, 2004) implemented in Matlab 7.1 (The Mathworks, Inc.). To exclude that TEPs or somatosensory-evoked potentials (SEPs) due to muscle twitch could mask the ERD/ERS (Kalcher \& Pfurtscheller, 1995), the epoching started $500 \mathrm{~ms}$ after the last stimulus of each train. The remaining intertrain interval was cut into 28 epochs of 500 ms so that for each condition (Sham1, Sham2, and Real) a total of 1120 epochs were obtained (Figure 1). Epochs with excessively noisy EEG, eye-movement artifacts (blinks or saccades), or muscle artifacts were excluded from further analysis after visual inspection. Because of a low quality of the EEG recording, due to exceeding artifact contaminations, 3 subjects had to be excluded from the study prior to postprocessing.

For each subject, epoch, and condition, the power spectra were estimated for the $\alpha(8-12 \mathrm{~Hz})$ and $\beta(13-30 \mathrm{~Hz})$ frequency bands by means of a Fast Fourier transform (Hamming window; frequency resolution $=2 \mathrm{~Hz}$ ). The mean band power was then obtained by averaging the power values of the sweeps for each session. To quantify the EEG power changes induced by TMS $\left(\mathrm{ERPow}_{\mathrm{x}}\right), \mathrm{ERD} / \mathrm{ERS}$ were computed as follows:

$$
\begin{aligned}
\text { ERPow }_{\mathrm{x}}= & {\left[\left(\text { Pow }_{\mathrm{x}} \text { Interest }- \text { Pow }_{\mathrm{x}} \text { Reference }\right)\right.} \\
& \left.\left./ \text { Pow }_{\mathrm{x}} \text { Reference }\right)\right] \times 100
\end{aligned}
$$

According to this formula, a power increase in the frequency band during the period of interest $\left(\right.$ Pow $_{\mathrm{x}}$ Interest) relative to the reference period ( Pow $_{\mathrm{x}}$ Reference) is expressed as a positive value, while a power decrease is expressed as a negative value. Sham 1 was used as reference period, while Real and Sham 2 stimulation were considered as two periods of interest. To find out whether $\alpha$ or $\beta$ frequencies were modulated depending on the duration of the stimulation, each 10-min session was divided into two consecutive periods of $5 \mathrm{~min}$ each. To this aim, the responses to the 40 trains of TMS were halved in blocks of 20 trains (block 1-B1: trains 1 to 20; block 2-B2: trains 21 to 40) (Figure 1). Each block of each session was referred to the corresponding block of the reference period (B1- or B2-Sham1). Since TMSunspecific effects over the experiment have been excluded by referencing the subsequent session to Sham1, we will refer to Sham 2 as the Post-real session.

\section{MEP Analysis}

MEPs from the right ADM were analyzed to test whether a correlation could be found between $\alpha$ and/or $\beta$ modulation and peripheral responses. Since induced $\alpha$ - and $\beta$-band activity was calculated in between trains (not in between pulses of a train), only MEPs elicited by the first stimulus of each train during the Real stimulation were considered. To this end, all MEPs elicited by the first stimulus of each train were expressed in peak-to-peak amplitude. The first train was excluded from the analysis, since the ERD/ERS calculation started from the first intertrain interval. Thereafter, the MEPs were divided in two blocks, in analogy to the EEG data. Since the stimulation was performed at resting motor threshold and to better study the relation between cortical and peripheral responses, MEPs below $50 \mu \mathrm{V}$ were not excluded from the analysis.

\section{Statistical Analysis}

A separate analysis of variance (ANOVA) with repeated measures was performed for each of the two frequency bands of interest testing the factors Session (Real, Post-real), Block (B1, B2), Region [frontal (F3, Fz, F4), central (C3, Cz, C4), parietal (P3, $\mathrm{Pz}, \mathrm{P} 4)$ ], and Side [right (F4, C4, P4), midline (Fz, Cz, Pz), left (F3, C3, P3)]. The Huynh-Feldt $\varepsilon$ correction factor was applied where appropriate to compensate for possible effects of nonsphericity in the compared measurements. The correction factor reduces the degree of freedom of the usual $F$ test. In all conditions, the normal distribution was tested applying the KolmogorovSmirnov test (for all $p>.2$ ). Post-hoc tests were performed to investigate significant effects, applying the Bonferroni correction as appropriate in the case of multiple comparisons.

A paired-sample $t$ test was performed to compare MEPs elicited in the two blocks of stimulation (B1-Real, B2-Real). To verify whether there was any correspondence between the modulatory effects of rTMS on the amplitude of the MEPs and the modulatory effects of rTMS on the event-related synchronization, a Pearson's correlation $(p<.05)$ coefficient was calculated between the changes in the MEPs and in the event-related synchronization in each frequency band over C3 and P3. C3 was selected because of its location over MI, whereas P3 recorded high ERS values during the second block of Real stimulation.

\section{Results}

rTMS did not cause any of the participants to experience adverse effects. The individual resting motor threshold ranged from 44 to $74 \%$ of maximum stimulator output (mean $=59 \pm 8$ ).

High frequency stimulation of MI induced an increase in synchronization (i.e., ERS) over central and parietal regions both in the $\alpha$ - and $\beta$-frequency band. The strongest effect was found for the $\alpha$ band, with an increase in power that outlasted the end of the Real session. Considering the whole experiment, $20 \mathrm{~Hz}$ rTMS was unable to induce any desynchronization (i.e., ERD) of $\alpha$ and $\beta$ frequencies, neither during the Real nor the Post-real session. Detailed results are described in the next section for $\alpha$ and $\beta$ separately.

\section{$\alpha$ Band}

The statistical analysis performed on the $\alpha$ band revealed no significant main effects [Session: $F(1,9)=0.02, p>.5)$; Block: $F(1,9)=0.02, \quad p>.5 ;$ Region: $F(2,18)=0.25, \quad p>.5 ; \quad$ Side: $F(2,18)=1.99, p>.5]$. A significant interaction Session $\times$ Side $[F(2,18)=6.45, p<.01]$ emerged, revealing a difference in the Real session when comparing the left stimulated side $(18.77 \%)$ to midline $(10.87 \%)$ and to the right, nonstimulated side $(9.35 \%)$ (all $p<.05$ ). No difference was found between midline and right sides during the Real session ( $p>1)$, and there was no difference among the three sides during the subsequent $10 \mathrm{~min}$ (Post-real, left $=13.37 \%$, midline $=14.24 \%$, right $=15.10 \%, p>1)$. Thus, we found a highly specific $\alpha$ induction over the target area, but only during the Real session. This result indirectly proves the goodness of our sham condition, which was unable to unbalance the relation among the three cortical sides.

A significant four-way interaction [Session $\times$ Block $\times$ Region $\times$ Side, $F(4,36)=3.19, p<.05]$ also emerged, highlighting two modulatory patterns, one over the left motor site and the 
other over the posterior parietal sites. As shown by the scalp distribution maps (Figure 2A), during B1-Real synchronization peaked over $\mathrm{C} 3$ and F3, representing the hot-spot. During the following $5 \mathrm{~min}$ of Real session (B2-Real), $\alpha$ power further increased over the stimulated area, until reaching the highest values (C3: $\mathrm{B} 2-\mathrm{Real}=28 \%$ vs. power over C3 across all blocks, $p<.03$ ) with a concurrent spread of synchronization over ipsi- and contralateral parietal regions. During Post-real session, a gradual return to baseline level for the stimulated motor site was found (C3: B1-Post-real $=20 \%$ vs. B2-Post-real $=5 \%, p<.03$ ). Conversely, ipsi and contralateral parietal sites and, to a lesser extent, $\mathrm{C} 4$ showed a different modulatory pattern (Figure 2A). In particular, Pz and P4 showed the largest synchronization during B1-
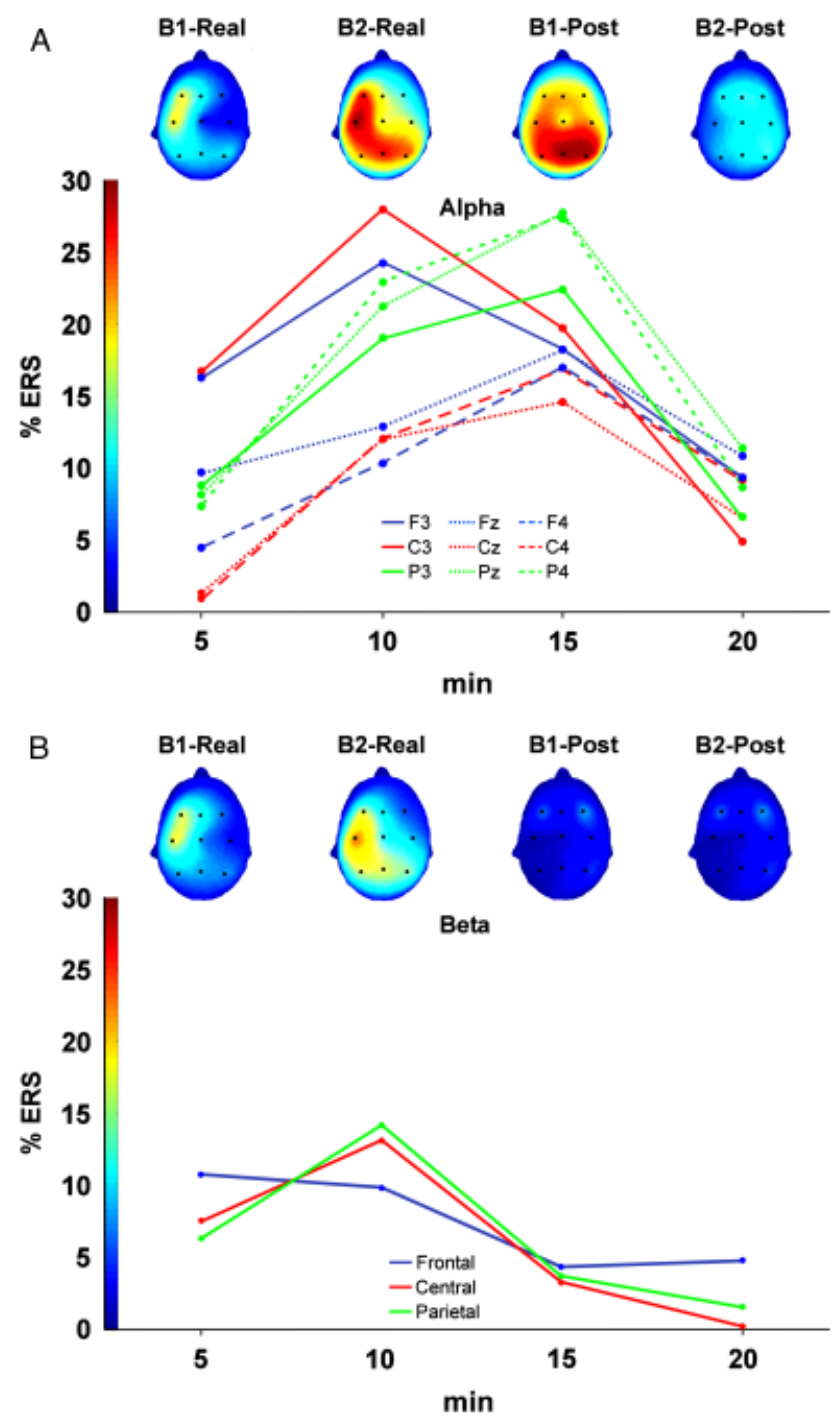

Figure 2. TMS effects in the $\alpha$-band (A) and $\beta$-band (B) as function of time. In the upper part of each figure (A and B), the scalp distribution maps of averaged event-related synchronization (ERS) induced by $20 \mathrm{~Hz}-$ TMS are shown. The four maps cover a period of $20 \mathrm{~min}(5 \mathrm{~min}$ bins), consisting of $10 \mathrm{~min}$ real stimulation (B1-Real, B2-Real) and the subsequent $10 \mathrm{~min}$ post-TMS interval (B1-Post-real, B2-Post-real). Voltage is color coded according to the color bar on the left. In the lower part, data are shown for each electrode and stimulation block for the $\alpha$ band (A), whereas for the $\beta$ band (B) data are plotted for each region and stimulation block. Note that all values are positive, since no ERD was found.
Post-real (all $p<.03$ ), which was also larger compared to all other recording sites in that block (B1-Post-real: $\mathrm{P} 3=22 \%$, $\mathrm{Pz}=28 \%, \mathrm{P} 4=28 \%$ vs. power over other electrodes, all $p<.001)$. During the last $5 \mathrm{~min}$ evaluated, no difference was found when comparing all scalp positions (all $p>.05$ ), since an overall return to baseline level was evident for all regions.

\section{$\beta$ Band}

The statistical analysis performed on the $\beta$-frequency band showed no significant main effects [Session: $F(1,9)=4.14$, $p>.05$; Block: $F(1,9)=0.059, p>.5$; Region: $F(2,18)=0.162$, $p>.5$; and Side: $F(2,18)=2.445, p>.5]$.

A significant interaction Session $\times$ Side $[F(2,18)=8.007$, $p<.005]$ indicated that synchronization over the left stimulated side was larger $(14 \%)$ when compared to midline $(8 \%)$ and to right side $(5 \%)$, but only during the Real session (all $p<.05$ ). Indeed, the Post-real period was characterized by low $\beta$ power values throughout (left side $=3 \%$, midline $=1 \%$, right $=5 \%$ ). Thus, similar to the $\alpha$ band, during the Real session the $\beta$-modulation was restricted over the targeted area.

The interaction Session $\times$ Block $\times$ Region $[F(2,18)=6.628, p<.005]$ proved that rTMS was able to modulate $\beta$ power over central and parietal regions, reaching their maximal synchronization during B2-Real (B2-Real: central region $=12 \%$ vs. power over the same region across all blocks, $p<.05$; parietal region $=13 \%$ vs. power over the same region across all blocks, $p<.01$ ) (Figure 2B). Interestingly, during the following $10 \mathrm{~min}, \beta$ synchronization underwent a rapid decrease (central region: $\mathrm{B} 1-$ Post-real $=3 \%$, B2-Post-real $=0.3 \%$; parietal region: $\mathrm{B} 1$-Post-real $=3 \%, \mathrm{~B} 2$-Post-real $=1 \%$ ). Despite frontal region reaching values similar to other regions, $\beta$ power did not show statistically significant modulation throughout the Real and Post-real stimulation session (ERS over frontal region: $\quad \mathrm{B} 1-$ Real $=10 \%, \quad \mathrm{~B} 2-\mathrm{Real}=9.1 \%, \quad \mathrm{~B} 1$-Post-real $=$ $4.05 \%$, B2- Post-real $=4.46 \% ; p>.09$ ).

\section{MEPs}

As can be seen in Figure 3A, the mean amplitude of the MEPs significantly increased in the second block of stimulation in comparison to the first block $[t(9)=-2.52, p<.03]$. To investigate the possible correlation between peripheral and cortical modulation, the Pearson coefficient was calculated between the changes in MEP amplitude and ERS values in the two blocks of the Real session (values recorded during B2, minus values recorded during $\mathrm{B} 1$ ). When considering the $\beta$ band, the increase in the MEP amplitude was inversely correlated with the increase of the power synchronization over C3 (Figure 3b). Indeed, a significant negative correlation emerged between $\beta$ power values and MEPs amplitude $(r=-0.65, p<.05)$. The $\beta$ synchronization was therefore greater in subjects in which small-amplitude MEPs were recorded.

No significant correlation was found between the increase of the MEPs and the increase of the synchronization amplitude over the P3 electrode. When considering the $\alpha$ band, no significant correlation emerged neither for C3 nor for P3. The same results emerged from Spearman's rank correlation analysis, indicating a significant correlation between $\beta$ values recorded over $\mathrm{C} 3$ and MEP amplitude $(r=-0.62, p<.05)$. 
A

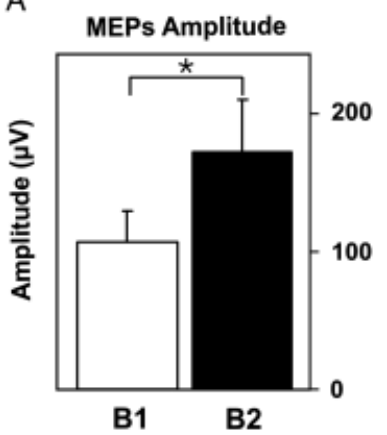

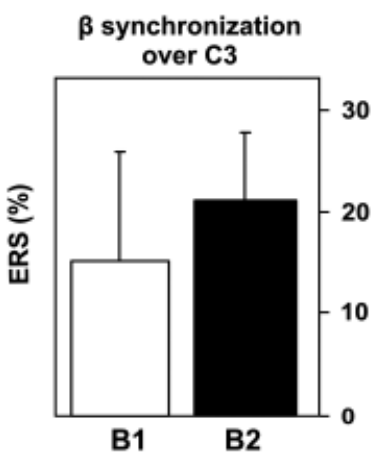

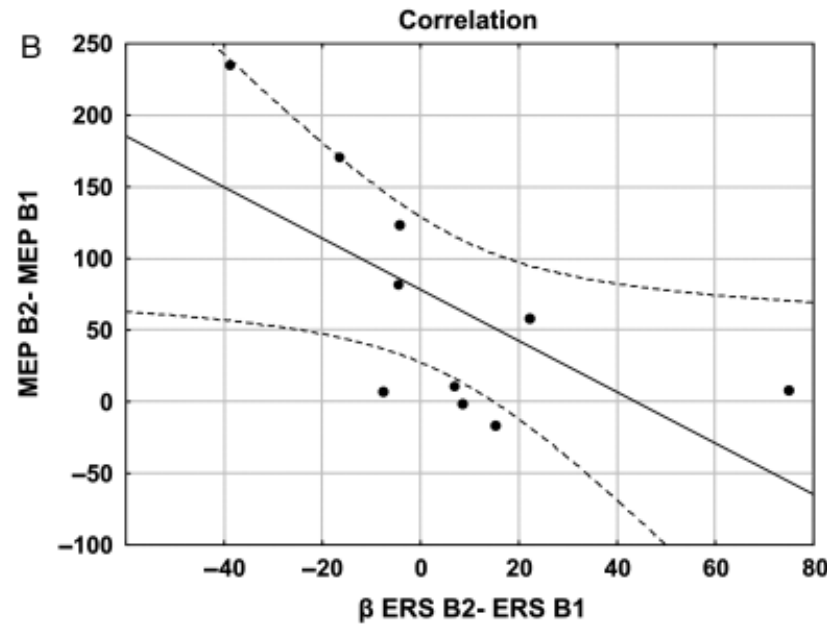

Figure 3. Motor-evoked potentials (MEP) amplitude and event-related synchronization (ERS) modulation. Panel A: Mean amplitude of the MEP (on the left) and of the power synchronization recorded over the electrode $\mathrm{C} 3$ (on the right) elicited during each block of Real stimulation (B1 and B2). Bars correspond to the standard error of mean, asterisk marks the significant difference between MEPs amplitude in the two blocks. Panel B: The scatterplot shows the significant negative correlation between the changes in the amplitude of the MEPs, on the $x$-axis and the changes in the power synchronization recorded over $\mathrm{C} 3$, on the $y$-axis. The dotted lines represent $95 \%$ confidence limits.

\section{Discussion}

The aim of the present study was to investigate EEG power modulations induced by high frequency TMS over MI. Specifically, we were interested in verifying if high frequency rTMS was able to induce a modulation in central $\alpha$ and/or $\beta$ power amplitude that was opposite to previous results obtained with low frequency rTMS (Brignani et al., 2008). Reproducing the effect of previous single pulse, $1 \mathrm{~Hz}$ or $5 \mathrm{~Hz}$ TMS studies (Brignani et al., 2008; Fuggetta et al., 2005, 2008; Paus et al., 2001), we found a widespread induced synchronization in both frequency bands, suggesting that rTMS outcome, as revealed by power variations in both bands, does not depend on the applied frequency.

The induced modulations were dose dependent for both bands, since the synchronization increased as a function of number of delivered stimuli, as shown by comparison of ERS values recorded during the first and second block of real TMS. Moreover, $\alpha$ induction outlasted the end of real stimulation, over a period of $5 \mathrm{~min}$. This suggests that rTMS at $20 \mathrm{~Hz}$ results in a progressively increasing modulatory effect, likely due to a temporal summation of the effects induced by each single pulse, able to bring to resonance the activity of a growing number of neurons of the targeted sensory-motor network. Speculatively, an external direct perturbation (e.g., TMS pulse) could cause a realignment of ongoing oscillatory activity, unmasking intrinsic oscillations and producing a commune cycle (Rosanova et al., 2009; Paus et al., 2001), where individual cells become synchronized by interacting with one another. It is noteworthy here that the mechanisms of bringing the stimulated area to resonate at its natural frequency independently of TMS protocol may be different from entrainment of oscillatory activity. Per definition, entrainment implies progressive phase-alignment of on-going oscillations to a rhythmic stream of external events, here the pulses of the TMS train. Consequently, entrainment would be expected to show a 1:1 frequency-locking between natural EEG-oscillations and the most effective rTMS-frequency promoting these oscillations (in our case $20 \mathrm{~Hz}$-TMS vis-à-vis promoting $\beta$-activity), which is inconsistent with $\alpha$ and $\beta$ coinduction over M1 across the many rTMS protocols tested $(1 \mathrm{~Hz}, 5 \mathrm{~Hz}, 20 \mathrm{~Hz})$. In addition, the few studies suggesting that it may be possible to bias performance by rTMS-induced entrainment of on-going oscillations showed this for performance during or immediately after the train (Romei, Driver, Schyns, \& Thut, 2011; Romei, Gross, \& Thut, 2010; Sauseng et al., 2009). Entrainment might therefore represent a very short-lasting phenomenon, and frequency specific $\beta$ entrainment to our $20 \mathrm{~Hz}$ trains might simply not have been detectable in the late and large time window we used for the analysis. It is also conceivable that $20 \mathrm{~Hz}$ rTMS is unable to enforce MI to oscillate in a rhythm that does not represent its intrinsic activity/state (Basar, Guntekin, \& Oniz, 2006) at rest, but would require specific conditions that are naturally characterized by activity in this frequency band, such as during a tonic muscle contraction (Hansen \& Nielsen, 2004).

A previous study by Fuggetta et al. (2008) found that central $\alpha$ synchronization after rTMS over MI was short lasting, restricted to $500 \mathrm{~ms}$ after the pulse, whereas no effect was found $2 \mathrm{~s}$ after the end of the train. The different frequency employed ( $5 \mathrm{~Hz}$ ) induced an $\alpha$ increase but with a different persistence (Houdayer et al., 2008). Nevertheless, it is likely that the effect of rTMS was underestimated by Fuggetta et al. (2008), since the ERD/ERS calculation was performed referring post-train induced power to a baseline period of $2 \mathrm{~s}$ before each train, that is, a moving reference interval was used. Therefore, as rTMS evolves and more and more synchronization is induced, there is also a progressive increase of power in the moving reference interval leading to an overcorrection of the TMS-induced cortical oscillatory activity.

We found that the increase of synchronization over time was associated with a concurrent change in topographic distribution. Indeed, over Real TMS to the Post-real TMS block, $\alpha$ and $\beta$ activities were found to spread from the targeted area towards posterior, parietal regions. Since TMS effects are strongest where the induced electric field is strongest (Rothwell, 1991), TMS is likely to affect activity in MI, which is supported by a maximal effect on $\alpha$ and $\beta$ activity over sites directly under the coil in the first block of real stimulation. This initial $\alpha$ response could find a physiological basis in recent papers, reporting that neocortical $\alpha$ can emerge from the activity of low threshold interneurons indicated as first responders when cortical excitatory activity (e.g., $20 \mathrm{~Hz}$ rTMS) increases (Fanselow, Richardson, \& Connors, 2008) or even from the intrinsic properties of layer V of pyramidal neurons (Bollimunta, Chen, Schroeder, \& Ding, 2008), which represents the "TMS target" in the MI cortex. In particular, it has been proposed that oscillatory activity around $10 \mathrm{~Hz}$ 
in the motor cortex emerges as a result of a large current sink (localized inward currents), which could be generated as a consequence of the magnetic pulse in the apical dendrites (I or II layer) with corresponding sources in layer V (Castro-Alamancos, Rigas, \& Tawara-Hirata, 2007). The spreading of activation from central to posterior regions is likely to follow the dense anatomical connections between motor and somatosensory cortex (sensory-motor areas). This would be in line with results from other EEG-TMS studies, in which synchronization was found to spread from stimulated cortex (C3) to ipsilateral parietal sites (P3, Pz) (Brignani et al., 2008; Fuggetta et al., 2008). Moreover, recent studies in humans provided strong evidence for the existence of interhemispheric pathways between posterior parietal cortex and contralateral MI (Koch et al., 2009; Zarei et al., 2006). Their mutual influence can be exerted directly through callosal connections and indirectly through the activation of pathways involving the homologous parietal regions (Koch et al., 2009). Both types of connection could explain the spreading of activation found from the central (i.e., MI) to posterior areas, ipsi and contralateral to the stimulation side.

We found that $\alpha$ and $\beta$ are differently modulated by TMS in terms of reactivity. A different magnitude of $\alpha$ and $\beta$ synchronization has been already reported during motor planning (Manganotti et al., 1998) and as a consequence of low and high frequency rTMS (Brignani et al., 2008; Fuggetta et al., 2008). Since the topographical distribution of ERD/ERS during different motor tasks suggests a relatively unspecific $\alpha$ change against a high somatotopic $\beta$ activation/deactivation (Crone et al., 1998; Pfurtscheller \& Lopes da Silva, 1999; Salmelin \& Hari, 1994), central $\alpha$ has been proposed to reflect a general activity of the motor area, whereas central $\beta$ has been proposed to be strongly associated with cortical control and monitoring of descending pathways (Baker, 2007; Jensen et al., 2005; Kilner, Baker, Salenius, Hari, \& Lemon, 2000; Salenius, Portin, Kajola, Salmelin, \& Hari, 1997). That TMS acts differentially on $\alpha$ and $\beta$ activity may be in line with these results. Since the motor cortex was in a resting condition and TMS represents an external perturbation (Rosanova et al., 2009), it is conceivable that the stimulation mainly affects the general activation of the stimulated area, represented by the $\alpha$ rhythm. In this vein, $\alpha$ could represent the natural frequency of primary motor cortex under resting conditions, which would be in line with in vitro studies (CastroAlamancos, 2000; Castro-Alamancos \& Rigas, 2002; CastroAlamancos et al., 2007). Regarding the $\beta$-band variations, we found no lasting effects, as already reported by Brignani et al. (2008), and a more focal topography if compared to the $\alpha$ spreading. The characteristics of $\beta$ modulations, restricted to the real stimulation, could be taken as evidence of its role as a cortical driver of spinal motoneuron activity, which is disengaged during a resting period (e.g., Post-real). The close relation between central $\beta$ and corticomuscular communication could thus explain its cessation after the end of the effective stimulation. This relation is also supported by the significant correlation between $\beta$ ERS and
MEP amplitudes. As reported by others (Lepage, SaintAmour, \& Theoret, 2008; Maki \& Ilmoniemi, 2010), $\beta$ synchronization was inversely related to MEPs amplitude, further corroborating the idea that an increase in central $\beta$ activity indexes a decreased excitability over motor area (Neuper et al., 2006). Despite being statistically significant, only a weak correlation between cortical and peripheral measurements emerged, probably because MEP size can be modulated by processes taking place at spinal level. Alternatively, as suggested by several TMS/ EEG studies (Lepage et al., 2008; Maki \& Ilmoniemi, 2010; Mitchell, Baker, \& Baker, 2007), brain oscillations are only limited predictors of MEPs size, in that EEG reflects the activity in a large cortical region, while the cortical activity related to MEPs control should be specific to the neurons controlling the target muscles. In particular, Mitchell et al. (2007), found that MEP amplitude is better predicted by EMG oscillations, which relates to the selected part of the cortical network projecting to the activated muscle.

A general consideration on the $\alpha$ power increase is that our data contradict the idea of opposite effects at cortical level of high and low frequency rTMS, which cannot be simply detected by opposite effects on $\alpha$ or $\beta$ power. Methodological limitations could account for this discrepancy. First, $\alpha$ or $\beta$ power modulations could not be good markers of rTMS outcome if we consider only the relationship between these indexes and stimulation frequency along with peripheral MEP responses, since other indexes have been shown to change as a consequence of opposite frequencies of stimulation. As an example, it has been reported that MI-rTMS at 1 and $5 \mathrm{~Hz}$ induces an increase or a decrease of intra and interhemispheric coherence, respectively (Oliviero, Strens, Di Lazzaro, Tonali, \& Brown, 2003; Strens et al., 2002). Similarly, opposite TMS-effects on brain activity have been found for several other EEG and ERP-measures and TMS over various sites (reviewed in Thut \& Pascual-Leone, 2010). It is also possible that a differential effect of low-high frequency rTMS could be better reflected by the modulation of other frequency bands, such as $\gamma$ or $\theta$ oscillations. This same reasoning could be applied to the relation between EEG power values and the classic MEP modulation after low and high frequency TMS. In particular, different indexes, such as corticomuscular coherence in $\beta$ band, could be more suitable to describe cortical control on efferent pathways (Mitchell et al., 2007).

In conclusion, we propose that $\alpha$ and $\beta$ induction represents a basic response of the human brain to TMS, as observed across many TMS protocols, at least for TMS over areas that generate $\alpha$ and $\beta$ such as the sensorimotor region. The long-lasting effect on central $\alpha$ power and its high reactivity suggest that this rhythm is related to TMS on resting motor cortex, whereas $\beta$ rhythm seems to be directly ascribed to motor cortex activation. This finding may have significance for understanding the intrinsic electrophysiological properties of the human cerebral cortex, which should be considered when using brain stimulation protocols that might aim to change cortical activity.

\section{References}

Baker, S. N. (2007). Oscillatory interactions between sensorimotor cortex and the periphery. Current Opinion in Neurobiology, 17, 649-655.

Basar, E., Guntekin, B., \& Oniz, A. (2006). Principles of oscillatory brain dynamics and a treatise of recognition of faces and facial expressions. Progress in Brain Research, 159, 43-62.
Bestmann, S., Ruff, C. C., Blankenburg, F., Weiskopf, N., Driver, J., \& Rothwell, J. C. (2008). Mapping causal interregional influences with concurrent TMS-fMRI. Experimental Brain Research, 191, 383-402.

Bollimunta, A., Chen, Y., Schroeder, C. E., \& Ding, M. (2008). Neuronal mechanisms of cortical alpha oscillations in awake-behaving macaques. Journal of Neuroscience, 28, 9976-9988. 
Bonato, C., Miniussi, C., \& Rossini, P. M. (2006). Transcranial magnetic stimulation and cortical evoked potentials: A TMS/EEG co-registration study. Clinical Neurophysiology, 117, 1699-1707.

Brignani, D., Manganotti, P., Rossini, P. M., \& Miniussi, C. (2008). Modulation of cortical oscillatory activity during transcranial magnetic stimulation. Human Brain Mapping, 29, 603-612.

Castro-Alamancos, M. A. (2000). Origin of synchronized oscillations induced by neocortical disinhibition in vivo. Journal of Neuroscience, 20, 9195-9206.

Castro-Alamancos, M. A., \& Rigas, P. (2002). Synchronized oscillations caused by disinhibition in rodent neocortex are generated by recurrent synaptic activity mediated by AMPA receptors. Journal of Physiology, 542, 567-581.

Castro-Alamancos, M. A., Rigas, P., \& Tawara-Hirata, Y. (2007). Resonance (approximately $10 \mathrm{~Hz}$ ) of excitatory networks in motor cortex: Effects of voltage-dependent ion channel blockers. Journal of Physiology, 578, 173-191.

Chen, R., Classen, J., Gerloff, C., Celnik, P., Wassermann, E. M., Hallett, M., \& Cohen, L. G. (1997). Depression of motor cortex excitability by low-frequency transcranial magnetic stimulation. Neurology, 48, 1398-1403.

Crone, N. E., Miglioretti, D. L., Gordon, B., Sieracki, J. M., Wilson, M. T., Uematsu, S., \& Lesser, R. P. (1998). Functional mapping of human sensorimotor cortex with electrocorticographic spectral analysis. I. Alpha and beta event-related desynchronization. Brain, 121, 2271-2299.

Delorme, A., \& Makeig, S. (2004). EEGLAB: An open source toolbox for analysis of single-trial EEG dynamics including independent component analysis. Journal of Neuroscience Methods, 134, 9-21.

Fanselow, E. E., Richardson, K. A., \& Connors, B. W. (2008). Selective, state-dependent activation of somatostatin-expressing inhibitory interneurons in mouse neocortex. Journal of Neurophysiology, 100, 2640-2652.

Ferreri, F., Pasqualetti, P., Maatta, S., Ponzo, D., Ferrarelli, F., Tononi, G., . . Rossini, P. M. (2010). Human brain connectivity during single and paired pulse transcranial magnetic stimulation. NeuroImage, 54 , $90-102$.

Fitzgerald, P. B., Fountain, S., \& Daskalakis, Z. J. (2006). A comprehensive review of the effects of rTMS on motor cortical excitability and inhibition. Clinical Neurophysiology, 117, 2584-2596.

Fuggetta, G., Fiaschi, A., \& Manganotti, P. (2005). Modulation of cortical oscillatory activities induced by varying single-pulse transcranial magnetic stimulation intensity over the left primary motor area: A combined EEG and TMS study. NeuroImage, 27, 896-908.

Fuggetta, G., Pavone, E. F., Fiaschi, A., \& Manganotti, P. (2008). Acute modulation of cortical oscillatory activities during short trains of high-frequency repetitive transcranial magnetic stimulation of the human motor cortex: A combined EEG and TMS study. Human Brain Mapping, 29, 1-13.

Hansen, N. L., \& Nielsen, J. B. (2004). The effect of transcranial magnetic stimulation and peripheral nerve stimulation on corticomuscular coherence in humans. Journal of Physiology, 561, 295-306.

Hari, R., \& Salmelin, R. (1997). Human cortical oscillations: A neuromagnetic view through the skull. Trends in Neuroscience, 20, 44-49.

Harris, I. M., \& Miniussi, C. (2003). Parietal lobe contribution to mental rotation demonstrated with rTMS. Journal of Cognitive Neuroscience, $15,315-323$.

Houdayer, E., Degardin, A., Cassim, F., Bocquillon, P., Derambure, P., \& Devanne, H. (2008). The effects of low- and high-frequency repetitive TMS on the input/output properties of the human corticospinal pathway. Experiments in Brain Research, 187, 207-217.

Jensen, O., Goel, P., Kopell, N., Pohja, M., Hari, R., \& Ermentrout, B. (2005). On the human sensorimotor-cortex beta rhythm: Sources and modeling. NeuroImage, 26, 347-355.

Kalcher, J., \& Pfurtscheller, G. (1995). Discrimination between phaselocked and non-phase-locked event-related EEG activity. Electroencephalography and Clinical Neurophysiology, 94, 381-384.

Kilner, J. M., Baker, S. N., Salenius, S., Hari, R., \& Lemon, R. N. (2000). Human cortical muscle coherence is directly related to specific motor parameters. Journal of Neuroscience, 20, 8838-8845.

Koch, G., Ruge, D., Cheeran, B., Fernandez Del Olmo, M., Pecchioli, C. Marconi, B., ... Rothwell, J. C. (2009). TMS activation of interhemispheric pathways between the posterior parietal cortex and the contralateral motor cortex. Journal of Physiology, $587,4281-4292$.
Komssi, S., \& Kahkonen, S. (2006). The novelty value of the combined use of electroencephalography and transcranial magnetic stimulation for neuroscience research. Brain Research Review, 52, 183-192.

Komssi, S., Kahkonen, S., \& Ilmoniemi, R. J. (2004). The effect of stimulus intensity on brain responses evoked by transcranial magnetic stimulation. Human Brain Mapping, 21, 154-164.

Komssi, S., Savolainen, P., Heiskala, J., \& Kahkonen, S. (2007). Excitation threshold of the motor cortex estimated with transcranial magnetic stimulation electroencephalography. NeuroReport, 18, 13-16.

Lepage, J. F., Saint-Amour, D., \& Theoret, H. (2008). EEG and neuronavigated single-pulse TMS in the study of the observation/ execution matching system: Are both techniques measuring the same process? Journal of Neuroscience Methods, 175, 17-24.

Macdonell, R. A., Shapir, B. E., Chiappa, K. H., Helmers, S. L., Cros, D., Day, B. J., \& Shahani, B. T. (1991). Hemispheric threshold differences for motor evoked potentials produced by magnetic coil stimulation. Neurology, 41, 1441-1444.

Maeda, F., Keenan, J. P., Tormos, J. M., Topka, H., \& Pascual-Leone, A. (2000). Modulation of corticospinal excitability by repetitive transcranial magnetic stimulation. Clinical Neurophysiology, 111, $800-805$.

Maki, H., \& Ilmoniemi, R. J. (2010). EEG oscillations and magnetically evoked motor potentials reflect motor system excitability in overlapping neuronal populations. Clinical Neurophysiology, 121, 492-501.

Manganotti, P., Gerloff, C., Toro, C., Katsuta, H., Sadato, N., Zhuang, P., ... Hallett, M. (1998). Task-related coherence and task-related spectral power changes during sequential finger movements. Electroencephalography and Clinical Neurophysiology, 109, 50-62.

Miniussi, C., Cappa, S. F., Cohen, L. G., Floel, A., Fregni, F., Nitsche, M. A., ... Walsh, V. (2008). Efficacy of repetitive transcranial magnetic stimulation/transcranial direct current stimulation in cognitive neurorehabilitation. Brain Stimulation, 1, 326-333.

Miniussi, C., \& Thut, G. (2010). Combining TMS and EEG offers new prospects in cognitive neuroscience. Brain Topography, 22, 249-256.

Mitchell, W. K., Baker, M. R., \& Baker, S. N. (2007). Muscle responses to transcranial stimulation in man depend on background oscillatory activity. Journal of Physiology, 583, 567-579.

Neuper, C., Wortz, M., \& Pfurtscheller, G. (2006). ERD/ERS patterns reflecting sensorimotor activation and deactivation. Progressive Brain Research, 159, 211-222.

Oliviero, A., Strens, L. H., Di Lazzaro, V., Tonali, P. A., \& Brown, P. (2003). Persistent effects of high frequency repetitive TMS on the coupling between motor areas in the human. Experimental Brain Research, 149, 107-113.

Pascual-Leone, A., Valls-Sole, J., Wassermann, E. M., \& Hallett, M. (1994). Responses to rapid-rate transcranial magnetic stimulation of the human motor cortex. Brain, 117, 847-858.

Paus, T. (2005). Inferring causality in brain images: A perturbation approach. Philosophical Transactions of the Royal Society London B: Biological Sciences, 360, 1109-1114.

Paus, T., Sipila, P. K., \& Strafella, A. P. (2001). Synchronization of neuronal activity in the human primary motor cortex by transcranial magnetic stimulation: An EEG study. Journal of Neurophysiology, 86, 1983-1990.

Pfurtscheller, G., \& Aranibar, A. (1977). Event-related cortical desynchronization detected by power measurements of scalp EEG. Electroencephalography and Clinical Neurophysiology, 42, 817-826.

Pfurtscheller, G., \& Lopes da Silva, F. H. (1999). Event-related EEG/ MEG synchronization and desynchronization: Basic principles. Clinical Neurophysiology, 110, 1842-1857.

Romei, V., Driver, J., Schyns, P. G., \& Thut, G. (2011). Rhythmic TMS over parietal cortex links distinct brain frequencies to global versus local visual processing. Current Biology, 21, 334-337.

Romei, V., Gross, J., \& Thut, G. (2010). On the role of prestimulus alpha rhythms over occipito-parietal areas in visual input regulation: Correlation or causation? Journal of Neuroscience, 30, 8692-8697.

Rosanova, M., Casali, A., Bellina, V., Resta, F., Mariotti, M., \& Massimini, M. (2009). Natural frequencies of human corticothalamic circuits. Journal of Neuroscience, 29, 7679-7685.

Rossi, S., Ferro, M., Cincotta, M., Ulivelli, M., Bartalini, S., Miniussi, C., ... Passero, S. (2007). A real electro-magnetic placebo (REMP) device for sham transcranial magnetic stimulation (TMS). Clinical Neurophysiology, 118, 709-716.

Rossi, S., Hallett, M., Rossini, P. M., \& Pascual-Leone, A. (2009). Safety, ethical considerations, and application guidelines for the use 
of transcranial magnetic stimulation in clinical practice and research. Clinical Neurophysiology, 120, 2008-2039.

Rossini, P. M., Barker, A. T., Berardelli, A., Caramia, M. D., Caruso, G., Cracco, R. Q., ... Tomberg, C. (1994). Non-invasive electrical and magnetic stimulation of the brain, spinal cord and roots: Basic principles and procedures for routine clinical application. Report of an IFCN committee. Electroencephalography and Clinical Neurophysiology, 91, 79-92.

Rothwell, J. C. (1991). Physiological studies of electric and magnetic stimulation of the human brain. Electroencephalography and Clinical Neurophysiology, 43(Suppl), 29-35.

Salenius, S., Portin, K., Kajola, M., Salmelin, R., \& Hari, R. (1997). Cortical control of human motoneuron firing during isometric contraction. Journal of Neurophysiology, 77, 3401-3405.

Salmelin, R., \& Hari, R. (1994). Spatiotemporal characteristics of sensorimotor neuromagnetic rhythms related to thumb movement. Neuroscience, 60, 537-550.

Sauseng, P., Klimesch, W., Heise, K. F., Gruber, W. R., Holz, E., Karim, A. A., . . . Hummel, F. C. (2009). Brain oscillatory substrates of visual short-term memory capacity. Current Biology, 19, 18461852.

Siebner, H. R., Bergmann, T. O., Bestmann, S., Johansen-Berg, H., Mochizuki, H., Bohning, D. E., ... Rossini, P. (2009). Consensus paper: Combining transcranial stimulation with neuroimaging. Brain Stimulation, 2, 58-80.
Strens, L. H., Oliviero, A., Bloem, B. R., Gerschlager, W., Rothwell, J. C., \& Brown, P. (2002). The effects of subthreshold $1 \mathrm{~Hz}$ repetitive TMS on cortico-cortical and interhemispheric coherence. Clinical Neurophysiology, 113, 1279-1285.

Thut, G., \& Miniussi, C. (2009). New insights into rhythmic brain activity from TMS-EEG studies. Trends in Cognitive Science, 13, 182-189.

Thut, G., \& Pascual-Leone, A. (2010). A review of combined TMS-EEG studies to characterize lasting effects of repetitive TMS and assess their usefulness in cognitive and clinical neuroscience. Brain Topography, 22, 219-232.

Triggs, W. J., Calvanio, R., Macdonell, R. A., Cros, D., \& Chiappa, K. H. (1994). Physiological motor asymmetry in human handedness: Evidence from transcranial magnetic stimulation. Brain Research, 636, 270-276.

Veniero, D., Bortoletto, M., \& Miniussi, C. (2009). TMS-EEG coregistration: On TMS-induced artifact. Clinical Neurophysiology, $120,1392-1399$.

Zarei, M., Johansen-Berg, H., Smith, S., Ciccarelli, O., Thompson, A. J., \& Matthews, P. M. (2006). Functional anatomy of interhemispheric cortical connections in the human brain. Journal of Anatomy, 209, 311-320.

(ReCEIVED November 8, 2010; ACCEPTED March 30, 2011) 